$\xi=-1$

\title{
Certification of the Rough Resonance by the Wave Site of Matter for Welter Point on the Alteration Recognition System
}

\author{
Jeong-lae $\mathrm{Kim}^{1}$, Kyu-dong $\mathrm{Kim}^{2} *$ \\ ${ }^{1}$ Department of Biomedical Engineering Eulji University, Seongnam, 13135, Korea \\ ${ }^{2}$ Department of Medical IT, Eulji University, Seongnam, 13135, Korea \\ *Corresponding authorE-mail: kdkim@eulji.ac.kr
}

\begin{abstract}
Wave alteration technology is formatted the resonance status for point-star pattern of the sparkle recognition rate (SRR) and vacuum recognition rate (VRR) on the wave recognition gestalt. The recognition rate condition by the wave recognition gestalt is associated with the welter resonance system. As to investigate a point-star pattern of the rough alteration, we are formatted of the wave value with wave layer point by the welter-down structure. The concept of recognition rate is made sure of the reference of sparkle rate and vacuum rate for alteration signal by the wave resonance gestalt. Moreover to appear a rough alteration of the SRR-VRR of the medium in terms of the wave-resonance gestalt, and wave point resonance that is gained the a wave value of the far alteration of the Wa-rm-FA- $\sigma_{\text {MEX-MED }}$ with $12.03 \pm 2.28$ units, that was the a wave value of the convenient alteration of the Wa-rm-CO- $\sigma_{\text {MEX-MED }}$ with $2.71 \pm 0.51$ units, that was the a wave value of the flank alteration of the Wa-rm-FL- $\sigma_{\text {MEX-MED }}$ with $1.41 \pm 0.19$ units, that was the a wave value of the vicinage alteration of the Wa-rm-VI- $\sigma_{\text {MEX-MED }}$ with $0.22 \pm 0.10$ units. The welter resonance will be to assess at the ability of the wave-resonance gestalt for the control degree recognition rate on the SRR-VRR that is clarified the rough sparkle and vacuum gestalt by the recognition rate system. Welter recognition system will be conjecture of a gestalt by the special signal and to count a wave data of welter resonance rate.
\end{abstract}

Keywords: sparkle recognition rate, wave recognition gestalt, welter recognition system, welter resonance.

\section{Introduction}

Matter variation comes from the wavelength moving and means spreading part. From the mathematical point of view, it is a complex mechanical figure and it is difficult to state if it is a point, a plane or it has minute dimension, and what its scatter observation is. A variation object is characterized by its regularity and repeatability, even if it is not visible for a human eye. The rate of this regularity is described by the variation dimension, which is not a positive integer number. The variation dimension does depend on the state of the object; meaning that any chief ingredient of the object looks exactly the same like the whole object. The variation shape describes the diversity of the object by evaluating the rapidity by which the matter degree, the surface area or the state of the object changes, with an increasing accuracy [1]. This is an important distinction because all methods for calculating variation dimension are applicable to self-dispersion versions. Specifically, it has revealed that the scattering method yields anomalously variable results for the variation dimension [2-3]. Another calculating the variation dimensions is to note that there are variable methods for calculating layer structure dimensions. The layer structure type are to reveal the divider method and stochastic methods [4].

In this study was the resonance status of the wave recognition technology that is formatted the rough alteration of the matter for point-star pattern with sparkle and vacuum alteration by the wave recognition gestalt. This sparkle and vacuum value is exhibited the sparkle rate (SR) and vacuum rate (VR) with the recognition function that is clarified to take a basis reference from wave layer, is revealed a position of the point-star pattern, check out the wave value with welter-down layer on the matter. The wave-resonance is to check out the ability of the alteration function with the rough degree that is accumulated the sparkle recognition rate and vacuum recognition rate by the wave recognition gestalt.

\section{Material and Method}

The wave recognition gestalt (Wa-RG) is appeared the peculiarity of point-star gestalt on the matter. Welter down layer position activity is analogized the rough changes by the sparkle down rate (SDR). The results of SDR are mediated to be the restriction of wave resonance rate (Wa-RR). The wave resonance gestalt (Wa$\mathrm{RG}$ ) is formatted of with matter of the wave resonance change in the sparkle activity and vacuum activity (Fig. 1)[5-6].

\subsection{Methods of Welter down Layer Position System}

The Wa-RG system is made use of the serious formation on the wave recognition gestalt system (Wa-RGS). Serious of Wa-RG is made use of the rough welter rate that is similar to a control waveresonance by welter down layer position technology (WDLPT). Rough wave resonance is formatted in the welter point gestalt that is induced by the wave layer (Wa-L) tool. The arithmetic peculiarity by Wa-RG is induced to the point of output-restrictions by the wave structure (Wa-S) in the welter point gestalt. The waveresonance gestalt by Wa-RG is made use of to the point of outputrestrictions by the welter recognition rate (WRR) in the Wa-RGS. The welter point gestalt (WPG) was estimated a down resonance technology (DRT) of side direction from welter down layer (WDL) on the WDLPT of Wa-RG. The welter recognition rate gestalt 
(WRRG) is to take welter signal from welter layer structure mechanisms on the WDLPT of Wa-RG. The wave sparkle vacuum rate (WSVR) is to take the welter recognition and the welter gestalt on WRR. The WRR is revealed to counter on the rough welter signal by the welter recognition gestalt (WRG) (Fig. 1)[7-8].

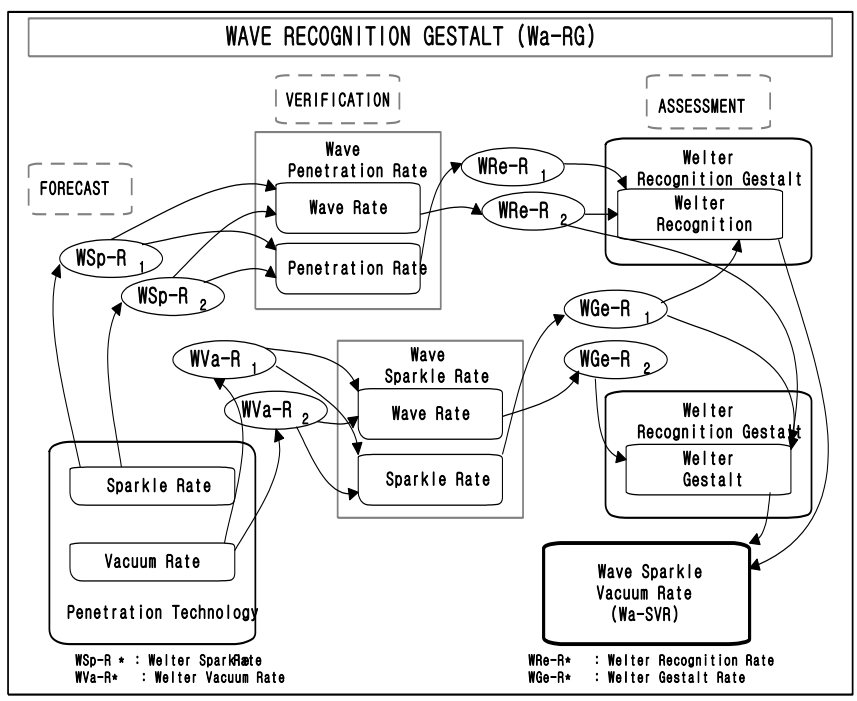

Fig. 1: System block of welter down layer position technology by sparkle rate and vacuum rate on the wave structure

\subsection{Stability Evaluation of Welter-Down Index}

Appear the welter-down site score on the Wa-RG is appeared with the Overall Resonance Rate (OVR), Far-Convenient Resonance Rate (FCRR) and Flank-Vicinage Resonance Rate (FVRR). These rates of standard deviations that are apprised the path of point around the side layer from the welter-down layer of the site and are make use of in degrees. The Wa-RG resonance rate scores are to take the displacement for rough signal in far-convenient (FC) and flank-vicinage (FV) that to reveal the Wa-FC and Wa-FV. The displacements at upper of layer from FC-axes of horizontal along Wa-FC as $\mathrm{x}$-direction and from $\mathrm{FV}$-axes of vertical Wa-FV along FV-axes as y-direction are revealed as Wa-RG-FC and WaRG-FV respectively. FCRR can check out that the phase of the main layer signal depends both on the propagation channel and the modulating properties of the side layer, which can be both frequency and power-dependent by the Wa-RG-FC. FVRR can make use of both amplitude and phase of the disclosed welter structure signal as $\mathrm{I}$ and $\mathrm{Q}$ is the current the far-convenient and flankvicinage by the Wa-RG-FV. Wa-FC is the modulated carrier of far-convenient on the Wa-RG, Wa-FV is the modulated carrier of flank-vicinage on the Wa-RG, $\Delta \mathrm{P}_{\mathrm{Wa}-\mathrm{RG}}$ is with amplitude and phase of the received welter structure signal of the $\mathrm{I}_{\mathrm{Wa}-\mathrm{FC}}$ and $\mathrm{Q}_{\mathrm{Wa}}$ Fv on the Wa-RG [9-10].

$\Delta \mathrm{P}_{\mathrm{Wa}-\mathrm{RG}}=\frac{\mathrm{I}_{\mathrm{Wa}-\mathrm{RG}-\mathrm{FC}}^{2}+\mathrm{Q}_{\mathrm{Wa}-\mathrm{RG}-\mathrm{FV}}^{2}}{\mathrm{Z}_{0}}, \varphi=\arctan \frac{\mathrm{Q}_{\mathrm{Wa}-\mathrm{RG}-\mathrm{FV}}}{\mathrm{I}_{\mathrm{Wa}-\mathrm{RG}-\mathrm{FC}}}(1)$

$\left|\Delta_{\gamma}\right|=\sqrt{\mathrm{I}_{\mathrm{Wa}-\mathrm{RG}-\mathrm{FC}}^{2}+\mathrm{Q}_{\mathrm{Wa}-\mathrm{RG}-\mathrm{FV}}^{2}}=$

$\sqrt{\Delta \mathrm{P}_{\mathrm{Wa}-\mathrm{RG}-\mathrm{RF}-\mathrm{FC}}+\mathrm{Z}_{0}}$

Where, $Z_{0}$ is the input impedance of the receiver. The indirectly measured welter-down site score data, represented as $\Delta \gamma$, is related to the differential reflection coefficient Wa-RG-FC and Wa-RG$\mathrm{FV}$, can thus be gained as:

$$
\angle\left(\Delta_{\gamma}\right)=\arctan \frac{\mathrm{Q}_{\mathrm{Wa}-\mathrm{RG}-\mathrm{FV}}}{\mathrm{I}_{\mathrm{Wa}-\mathrm{RG}-\mathrm{FC}}}=\varphi
$$

Therefore, the test setting that includes the communication range between pin of wave resonance layer and their system consist of the properly appear by the monitoring [11].
Welter welter-down gestalt (We-DLG) is check out a combination scores both We-DLG-FV and We-DLG-FC on the wave resonance layer. The "We-DLG-value" is to take from absolute $\sigma-W a-R G$ values, so it is more sensitive to FV-FC and $\sigma$-Wa-RG level fluctuations. In general, the $\sigma$-Wa-RG based We-DLG makes use of the free space propagation model in Eq. 4 :

$\sigma-W a-R G(r)[n . u]=.\sigma$-We-DLG-FC $\gamma / \mathrm{r}^{\sigma-W e-D L G-F V ~} \equiv \sigma$-Wa-RG(r)[dB]
$=20 \log 10(\sigma$-We-DLG-FV $)-\sigma$-We-DLG-FC $20 \log 10(\mathrm{r})$

' $\mathrm{r}$ ' is the range or distance, and $\sigma$-We-DLG-FV and $\sigma$-We-DLG-FC are coefficients that can be apprised from a non-linear regression that minimizes the root mean square (RMS) by a set of between wave resonance layer. The expression rate of $\sigma-\mathrm{Wa}-\mathrm{RG}(\mathrm{r})$ is already linear with respect to $\sigma$-We-DLG-FV and $\sigma$-We-DLG-FC [12-13][30].

\section{Results and Discussion}

\subsection{Properties of the Sequence Selection}

Wave recognition gestalt (Wa-RG) is checked out the resonance status for point-ring pattern of the sparkle rate (SR) and vacuum rate (DR) on the resonance technology (RT) condition. RT is to fix the rough objects of the wave sparkle rate (Wa-SR) on the WaRG-gestalt. And, RT is to embezzle the equivalent things of the wave vacuum rate (Wa-VR) on the Wa-RG-gestalt. The results are ascertained the wave recognition gestalt system (Wa-RGS) in accordance with the restriction of sparkle recognition rate (SRR). The experiment is lead to peculiar an alteration of vacuum recognition rate (VRR) is appeared in the welter recognition gestalt activities (WRGA). The experiment of Wa-RG-gestalt is revealed the Wa-rg- $\sigma_{\text {MED }}$, Wa-rg- $\sigma_{\text {AVG }}$ and Wa-rg- $\sigma_{\text {MAX-AVG }}$ database which are collected from the wave signal resonance gestalt by the Wa-rg activities (Table 1). Wave signal resonance gestalt data are used Matlab6.1 for the calculations.

\subsection{Improvements of Multiple Sequence Selections}

Comparison Database of SRR-VRR on the Wa-rg- $\sigma_{\text {MED }}$ and Wa$\mathrm{rg}-\sigma_{\mathrm{AVG}}$ and Wa-rg- $\sigma_{\mathrm{MAV}-\mathrm{AVG}}$ :

Wave recognition gestalt (Wa-RG) on the far $(F A-\sigma)$ condition is to be appeared a sparkle recognition rate-vacuum recognition rate (SRR-VRR) value for the Wa-rg-FA- $\sigma_{\mathrm{MED}}$, Wa-rg-FA- $\sigma_{\mathrm{AVG}}$ and Wa-rg-FA- $\sigma_{\text {MAX-AVG }}$ (Fig. 2). The large wave of the Wa-rg-FA$\sigma_{\text {MED }}$ is to the flank-vicinage (FV) direction in the Wa-RGS. Furthermore, Wa-rg activities of far SRR-VRR are ascertained the small wave to contrast between the Wa-rg-FA- $\sigma_{\mathrm{AVG}}$ and Wa-rgFA- $\sigma_{\text {MAX-AVG }}$ with the same direction in the Wa-RGS. In the Warg activities of far SRR-VRR is checked out a large wave at $13.37 \pm 1.48$ unit with Wa-rg-FA- $\sigma_{\text {MED }}$ of the wave structure gestalt. In the far SRR-VRR of Wa-rg activities is checked out large wave at $15.60 \pm 3.32$ unit with Wa-rg-FA- $\sigma_{\mathrm{AVG}}$ in the Wa-RGS. The activities of wave structure gestalt in the far SRR-VRR is to be take that a wave mediate is arise the FV direction in the Wa-RGS. It is a rough role in the wave activities of a Wa-rg-Far of far resonance. In the wave of Wa-rg activities is checked out a small wave at $9.81 \pm 3.43$ unit with Wa-rg-FA- $\sigma_{\text {MAX-AVG. The welter phenome- }}$ non of the far SRR-VRR is lead serious to vary the Wa-RGS by the welter structure in the Wa-rg activities direction. Wave recognition gestalt (Wa-RG) of convenient $(\mathrm{CO}-\sigma)$ condition is to be appeared a sparkle recognition rate-vacuum recognition rate (SRR-VRR) value for the Wa-rg-CO- $\sigma_{\mathrm{MED}}$, Wa-rg-CO- $\sigma_{\mathrm{AVG}}$ and Wa-rg-CO- $\sigma_{\text {MAX-AVG }}$ (Fig. 2). Wa-rg activities of convenient SRRVRR are ascertained the some wave to contrast between Wa-rg$\mathrm{CO}-\sigma_{\mathrm{MED}}$ and Wa-rg-CO- $\sigma_{\mathrm{AVG}}$ with the same direction in the WaRGS. 
Table 1: Average of the wave structure gestalts: the far SRR-VRR (Warg-FA $\sigma_{\text {MAX-AVG }}$ ), convenient SRR-VRR (Wa-rg-CO $\sigma_{\text {MAX-AVG }}$ ), flank SRRVRR (Wa-rg-FL $\sigma_{\text {MAX-AVG }}$ ) and vicinage SRR-VRR (Wa-rg-VI $\sigma_{\text {MAX-AVG }}$ ) condition. Average of Wa-rg- $\sigma_{\mathrm{MAX}}$ and Wa-rg- $\sigma_{\mathrm{AVG}}$

\begin{tabular}{|c|c|c|c|c|}
\hline Average $\sigma$ & $\begin{array}{c}\text { FA } \sigma_{\text {Avg-SRR- }} \\
\text { VRR }\end{array}$ & $\begin{array}{c}\text { CO } \sigma_{\text {Avg- }} \\
\text { SRR-VRR }\end{array}$ & $\begin{array}{c}\text { FL } \sigma_{\text {Avg-SRR- }} \\
\text { VRR }\end{array}$ & $\begin{array}{c}\text { VI } \sigma_{\text {Avg-SRR- }} \\
\text { VRR }\end{array}$ \\
\hline $\begin{array}{c}\text { Wa-rg- } \\
\sigma_{\text {MAX-AVG }}\end{array}$ & $17.40 \pm 5.02$ & $5.17 \pm 0.43$ & $2.27 \pm 0.29$ & $0.39 \pm 0.04$ \\
\hline $\begin{array}{c}\text { Wa-rg- } \\
\sigma_{\text {MAX-MED }}\end{array}$ & $12.03 \pm 2.28$ & $2.71 \pm 0.51$ & $1.41 \pm 0.19$ & $0.22 \pm 0.10$ \\
\hline
\end{tabular}

Whereas, the Wa-rg activities of convenient SRR-VRR is checked out small wave the Wa-rg-CO- $\sigma_{\mathrm{MAX}-\mathrm{AVG}}$ by the wave structure gestalt on the FV direction in the Wa-RGS. Wa-rg activities of convenient SRR-VRR are checked out small wave at $7.28 \pm 0.67$ unit with Wa-rg-CO- $\sigma_{\text {MED }}$ of the wave structure gestalt. In the convenient SRR-VRR of Wa-rg activities is checked out small at $7.36 \pm 0.57$ unit with Wa-rg-CO- $\sigma_{\mathrm{AVG}}$ on the $\mathrm{FC}$ direction in the Wa-RGS. The activities of wave structure gestalt in the convenient SRR-VRR is to be take that a wave is arise the same direction in the Wa-RGS. But, it is a rough role in the wave activities of a convenient resonance. In the wave of Wa-rg activities is checked out very small wave at $2.63 \pm 0.31$ unit with Wa-rg-CO- $\sigma_{\mathrm{MAX}-\mathrm{AVG}}$ on the FC direction. The welter phenomenon of the convenient SRR-VRR is lead serious to vary the Wa-RGS by the welter structure in the same direction. The convenient SRR-VRR is checked out to vary a very more alteration of welter resonance than the far SRR-VRR in the Wa-rg activities direction. Wave recognition gestalt (Wa-RG) of flank (FL- $\sigma$ ) condition is to be appeared a sparkle recognition rate-vacuum recognition rate (SRR-VRR) value for the Wa-rg-FL- $\sigma_{M E D}$, Wa-rg-FL- $\sigma_{\text {AVG }}$ and Wa-rg-FL$\sigma_{\text {MAX-AVG }}$ (Fig. 2). Wa-rg activities of flank SRR-VRR are ascertained small wave at Wa-rg-FL- $\sigma_{\mathrm{MED}}$ and Wa-rg-FL- $\sigma_{\mathrm{AVG}}$ of the wave structure gestalt on the FV direction in the Wa-RGS. Whereas, differently the very small wave value of Wa-rg-FL$\sigma_{\text {MAX-AVG }}$ is to the FV direction in the Wa-RGS. Wa-rg activities of flank SRR-VRR is checked out small wave at $2.38 \pm 0.30$ unit with Wa-rg-FL- $\sigma_{\text {MED }}$ of the wave structure gestalt. In the flank SRR-VRR of Wa-rg activities is checked out slightly small at $2.56 \pm 0.34$ unit with Wa-rg-FL- $\sigma_{\mathrm{AVG}}$ on the FC direction in the Wa-RGS. The activities of the wave structure gestalt in the flank SRR-VRR is to be take that a wave is arise the same direction in the Wa-RGS. But, it is a rough role in the wave activities of a flank resonance. In the wave of Wa-rg activities is checked out small wave at $1.22 \pm 0.16$ unit with Wa-rg-FL- $\sigma_{\text {MAX-Avg. The wel- }}$ ter phenomenon of the flank SRR-VRR is lead serious to vary the Wa-RGS by the welter structure in the same direction. The flank SRR-VRR is lead excellently to vary the Wa-RGS by the welter resonance at the Wa-rg activities. Wave recognition gestalt (Wa$\mathrm{RG}$ ) of vicinage (VI- $\sigma$ ) condition is to be appeared a sparkle recognition rate-vacuum recognition rate (SRR-VRR) value for the Wa-rg-VI- $\sigma_{\text {MED }}$, Wa-rg-VI- $\sigma_{\text {AVG }}$ and Wa-rg-VI- $\sigma_{M A X-A V G}$ (Fig. $2)$. Wa-rg activities of vicinage SRR-VRR are ascertained small wave at Wa-rg-VI- $\sigma_{\text {MED }}$ and Wa-rg-VI- $\sigma_{\text {AVG }}$ of the wave structure gestalt on the FC direction in the Wa-RGS. Whereas, differently the small wave value of Wa-rg-VI- $\sigma_{\mathrm{MAX}-\mathrm{AVG}}$ is to the normal direction in the Wa-RGS. Wa-rg activities of vicinage SRR-VRR is checked out very small wave at $0.46 \pm 0.01$ unit with Wa-rg-VI$\sigma_{\text {MED }}$ of the wave structure gestalt. In the vicinage SRR-VRR of Wa-rg activities is checked out very small at $0.48 \pm 0.06$ unit with Wa-rg-VI- $\sigma_{A V G}$ on the FC direction in the Wa-RGS. The activities of the wave structure gestalt in the vicinage SRR-VRR is to be take that a wave is arise the same direction in the Wa-RGS. But, it is a rough role in the wave activities of a vicinage resonance. In the wave of Wa-rg activities is checked out very small wave at $0.20 \pm 0.05$ unit with Wa-rg-VI- $\sigma_{\text {MAX-AVG }}$ on the FC direction in the Wa-RGS. The welter phenomenon of the vicinage SRR-VRR is lead serious to vary the WA-RGS by the welter structure in the normal direction. The vicinage SRR-VRR is lead slightly to vary the Wa-RGS by the welter resonance at the Wa-rg activities.

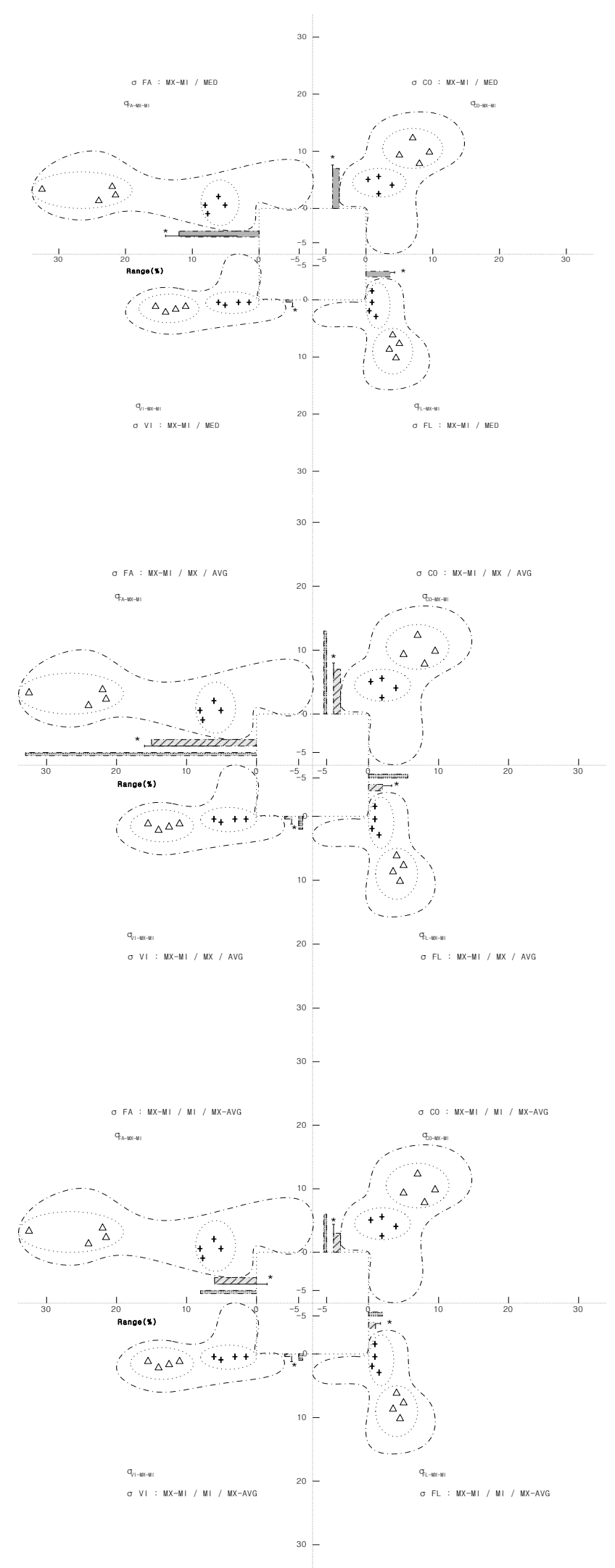

Fig. 2: Wa-rg-gestalt of the data on the wave condition for activities: restriction of the Wa-rg- $\sigma_{\mathrm{MED}}$ and Wa-rg- $\sigma_{\mathrm{AVG}}$ and Wa-rg- $\sigma_{\mathrm{MAX}-\mathrm{AVG}}$

\section{Conclusion}

In this paper, wave recognition technology was to format the resonance recognition with the wave recognition gestalt by the wave layer of recognition rate. This wave gestalt was to be exhibited a point of the wave-resonance by the recognition rate, to make sure 
of an alteration data from the basis reference by sparkle rate (SR) and vacuum rate (VR). As to investigate a position of the wave layer, we are checked out the wave point with welter-down layer on the matter distribution. Therefore, the wave-resonance is to check out the ability of the alteration function with the rough degree that is accumulated the sparkle recognition rate and vacuum recognition rate by the wave recognition gestalt.

\section{Acknowledgments}

This study is a revised and expanded version of a paper entitled "Feature of swell resonance of the alteration on the matter" presented at Smart Convergence of Culture Technology Letters with IICCC 2018, July 23-28, Ho Chi Minh\& DaNang, Vietnam.

\section{References}

[1] Omiotek Z. (2011), The study of the self-similarity measurement of the images by fractal analysis (in polish), Barometr Regionalny, 23(1), 93-105.

[2] Brown, S.R. (1987), A note on the description of surface roughness using fractal dimension. Geophys. Res. Lett., 14 (11), 1095-1098.

[3] Dannenberg R. (2004), Quantitative comparison of lineal analysis to box counting analysis of a real microtexture., Harmon Fract Image Anal, 50-60.

[4] Carr, J.R., Benzer, W.B. (1991), On the practice of estimating fractal dimension. Math. Geol., 23 (7), 945-958.

[5] Kim, K.D., Kim, J.L., Yu. Y.M. (2013), A Study on the Influence of Mobile Commerce Characteristics Perception on Mobile Shopping Intentions, The Journal of IIBC, 13(6), 297-303.

[6] Kim, J.L., Hwang, K.S. (2015), Study of quake wavelength of dynamic movement with posture, International Journal of Advanced Smart Convergence, 4(1), 99-103.

[7] Kim, J.L., Kim, K.D. ) (2017), Prediction of shiver differentiation by the form alteration on the stable condition, International Journa of Internet Broadcasting and Communication, 9(4), 8-13.

[8] Kim, J.L., Kim, H.J. (2017), A Study of structure modeling of the stratum corneum on the hydration, Journal of the Convergence on Culture Technology, 3(3), 31-36.

[9] Huiting J, Flisijn H, Kokkeler ABJ, Smit GJM. (2013), Exploiting phase measurements of EPC Gen2 RFID structures. IEEE Int Conf RFID-Technol Appl (RFID-TA), 1-6.

[10] Bekkali A, Zou SC, Kadri A, Crisp M, Penty RV. (2015), Performance analysis of passive UHF RFID systems under cascaded fading channels and interference effects. IEEE Trans Wirel Commun., 14(3), 1421-33.

[11] DiGiampaolo E, Martinelli F. (2014), Mobile robot localization using the phase of passive UHF RFID signals. IEEE Trans Ind Electron, 61(1), 365-76.

[12] López Y. Á., Gómez M.E., Andrés F.L.H. (2017), A received signal strength RFID-based indoor location system, Sensors and Actuators A 255, 118-133.

[13] Chawla K., McFarland C., Robins G., Shope C. (2013), Real-time RFID localization using RSS, in: 2013 International Conference on Localization and GNSS (ICL-GNSS), Turin (Italy), (25-27 June), $1-6$ 